\title{
Potential/complex-lamellar velocity decomposition and its relevance to turbulence
}

\author{
By RONALD L. PANTON \\ Mechanical Engineering Department, University of Texas, Austin
}

(Received 8 April 1977 and in revised form 28 February 1978)

In discussing turbulent shear layers, experimentalists have divided the flow into a turbulent region, which is vortical, and a non-turbulent region, which is irrotational but unsteady. This paper introduces a theoretical method of decomposing the velocity field into potential and vortical components that is compatible with the experimentalists' viewpoint. Specifically, only potential motions will exist in the nonturbulent region, while the decomposition shows that the turbulent region consists of both potential and vortical motions. The kinematic decomposition used is called a potential/complex-lamellar decomposition. Compared with the standard Helmholtz decomposition, the complex-lamellar decomposition is not widely known, and this article includes a discussion of its properties and characteristics. The vector components in this decomposition may be represented by three scalar potentials: $\phi, \psi$ and $\chi$. One of the important physical interpretations of the potentials concerns vortex lines. Vortex lines are defined by the intersection of a surface $\psi=$ constant with a surface $\chi=$ constant. Since these surfaces are a function of time, this establishes a sound kinematic theory for following the history of vortex lines in a turbulent or viscous flow.

\section{Introduction}

The turbulent/non-turbulent interface recognized by Corrsin (1943) separates a completely irrotational flow from the vortical flow. Experimentalists have identified many complex and interacting processes within the turbulent flow. These processes may increase or decrease length scales. They may produce, transfer or destroy quantities such as momentum, kinetic energy and vorticity. In any event, one of the major characteristics of these processes is that they occur within the turbulent zone and have a limited region of influence.

In order to explain turbulent processes, several investigators have decomposed the velocity field. Townsend's $(1976$, p. 105) decomposition emphasized large eddies as a dominant source of Reynolds stresses, while Hussain \& Reynolds (1970) and Landahl (1967) calculated a wave-like decomposition. More recently, Libby (1975) decomposed the velocity on the basis of whether the flow was turbulent or non-turbulent. This decomposition was inspired by the conditional-averaging techniques developed by Kovasznay, Kibens \& Blackwelder (1970). None of these decompositions have, or were intended to have, a kinematic basis.

A kinematic decomposition always separates the velocity field into a potential component and a rotational or vortical component. The traditional separation is 
usually called Helmholtz's (1857) decomposition, although some authors note that Stokes (1851) used a similar method at a prior date. In this paper, a different decomposition is used where the vortical component is complex lamellar. With this method the flow in the non-turbulent region becomes completely potential, while inside the turbulent region the flow becomes a mixture of potential and vortical components. That is, the complex-lamellar component is non-zero only when the vorticity is non-zero. The decomposition provides a firm mathematical basis for the idea that potential motions exist within the turbulent zone and that vortical motions are confined to the turbulent region.

Several investigators have discussed turbulence phenomena in terms of vortex lines and their motion. In a viscous flow there is a conceptual difficulty in following the history of vortex lines that is sometimes overlooked. One of the major results of the paper will show how the potential/complex-lamellar decomposition supplies the theoretical basis for discussing vortex-line history. In a certain sense this is the viscous complement to Helmholtz's theorem, which states that vortex lines follow the material particles in an inviscid flow.

A major section of the paper ( $\$ 3$ ) gives the general characteristics of the potential/ complex-lamellar decomposition. Next, the dynamic equations are investigated to see how the decomposition influences various terms in the equations. 'The last section discusses the characteristics of the decomposition when it is applied to the turbulent wall layer.

\section{Background}

The kinematic components of fluid motion are defined using only local considerations. The local instantaneous motion of one particle with respect to a neighbour can be divided into deformation and solid-body rotation. If the strain-rate tensor is $\mathbf{S}$ and the vorticity is $\omega=\nabla \times \mathbf{v}$, then two points a distance $d \mathbf{x}$ apart have a relative motion consisting of the two components

$$
d \mathbf{v}=d \mathbf{x} \cdot \mathbf{S}+\frac{1}{2} \omega \times d \mathbf{x}=d \mathbf{v}_{(\mathrm{g})}+d \mathbf{v}_{(r)}
$$

This expression gives a kinematic decomposition of $d \mathbf{v}$ which is precise as $d \mathbf{x}$ becomes small. However, this equation is useless if one tries to decompose the entire velocity field into a contribution due to rotational motions and a contribution due to straining motions. It is not possible to integrate (2.1) between two points in the flow and call the contribution of each integral on the right-hand side a component due to a particular motion. In general, the parts on the right-hand side are not exact differentials because the separate contributions depend upon the path chosen for integration.

The successful approach to decomposing the velocity field into kinematic parts is to take one component $\tilde{\alpha}$ as a potential flow. The necessary and sufficient condition for a potential to exist is $\nabla \times \tilde{\alpha}=0$. Therefore $\tilde{\alpha}$ contributes nothing to the vorticity while the other component $\tilde{\beta}$ contributes everything:

$$
\mathbf{v}=\tilde{\alpha}+\tilde{\beta}=\nabla \tilde{\phi}+\tilde{\beta} .
$$

Since $d \tilde{\boldsymbol{\alpha}}$ and $d \mathbf{v}$ are exact differentials, their difference $d \tilde{\boldsymbol{\beta}}$ must also be exact. The decomposition is not unique since any potential flow may be used for $\tilde{\alpha}$. 
Equation (2.2) confines the rotational motion to the $\tilde{\beta}$ component. A complete separation of deformation and rotation would be achieved if $\tilde{\beta}$ did not contribute to the strain rate, i.e. if $\nabla \tilde{\beta}$ were antisymmetric. In general, this condition is too stringent, and one must accept contributions to the strain rate from both $\tilde{\alpha}$ and $\tilde{\beta}$.

Two different methods of completing the decomposition will be discussed. In one method $\tilde{\beta}$ is required to be solenodial $(\nabla \cdot \tilde{\beta}=0)$ and in the second $\beta$ is required to be complex lamellar $(\beta . \nabla \times \beta=0)$.

Helmholtz's decomposition, which is the most well known and widely used, will be outlined first. It is constructed by further requiring that $\nabla \cdot \tilde{\beta}=0$, so that any sources are confined to the $\tilde{\alpha}$ component; $\tilde{\Delta}=\nabla . v=\nabla . \tilde{\alpha}$. As discussed in many textbooks (Phillips 1933; Brand 1947; Aris 1962; Batchelor 1967; Richardson \& Cornish 1977), $\tilde{\beta}$ may be represented by a vector potential $\mathbf{B}$ :

$$
\tilde{\boldsymbol{\beta}}=\nabla \times \mathbf{B} \text {. }
$$

A unique choice for $\mathbf{B}$ is made on purely mathematical grounds. The vector identity $\nabla^{2} \mathbf{B}=\nabla(\nabla . \mathbf{B})-\nabla \times(\nabla \times \mathbf{B})$ will become Poisson's equation, $\nabla^{2} \mathbf{B}=-\boldsymbol{\omega}$, if one requires that $\nabla . \mathbf{B}=0$. Then the Biot-Savart law is found as a solution for $\tilde{\boldsymbol{\beta}}$ :

$$
\tilde{\beta}=-\frac{1}{4 \pi} \int \frac{\mathbf{r} \times \omega^{\prime}}{|\mathbf{r}|^{3}} d V^{\prime}
$$

In (2.4), $\mathbf{x}$ is the point of evaluation of $\tilde{\beta}, \mathbf{x}^{\prime}$ the position of the element $d V^{\prime}$ and $\mathbf{r}=\mathbf{x}-\mathbf{x}^{\prime}$. The potential component $\tilde{\alpha}$ is similarly related to a distribution of sources since $\nabla^{2} \tilde{\phi}=\tilde{\Delta}$ :

$$
\tilde{\boldsymbol{\alpha}}=\nabla \Phi+\frac{1}{4 \pi} \int \frac{\mathbf{r} \Delta^{\prime}}{|\mathbf{r}|^{3}} d V^{\prime}
$$

The solutions depend upon only the internal distribution of vorticity and sources. A harmonic function $\Phi$ is added to $\delta$ as required to satisfy boundary conditions.

The fact that the scalar and vector potentials have been chosen to satisfy the Poisson equation allows one to prove that the decomposition has global validity. This is one advantage of Helmholtz's decomposition. This choice of potentials also results in the Biot-Savart equation for $\tilde{\boldsymbol{\beta}}$, and therefore attributes the influence of vorticity to remote positions. It is sometimes said that the vorticity induces the remote flow. This is not a dynamic result, but a mathematical consequence of requiring that $\nabla . \mathbf{B}=0$. In regions where the vorticity vanishes, $\tilde{\beta}$ will be non-zero and will be locally irrotational. Thus the existence of $\tilde{\boldsymbol{\beta}}$ in a region does not indicate that there is vorticity in that region. Dynamically, the propagation of vorticity is accomplished by diffusion and convection processes. The Helmholtz method divides the field in a kinematically consistent way without regard to dynamic processes.

\section{Potential/complex-lamellar decomposition}

Viscous turbulent processes are local in nature, thus one would like a decomposition where the external flow, including the unsteady motions in the non-turbulent region, is completely potential. In terms of the decomposition, this means that $\boldsymbol{\beta}$ should become zero when the vorticity becomes zero. The potential/complex-lamellar decomposition has this property. 


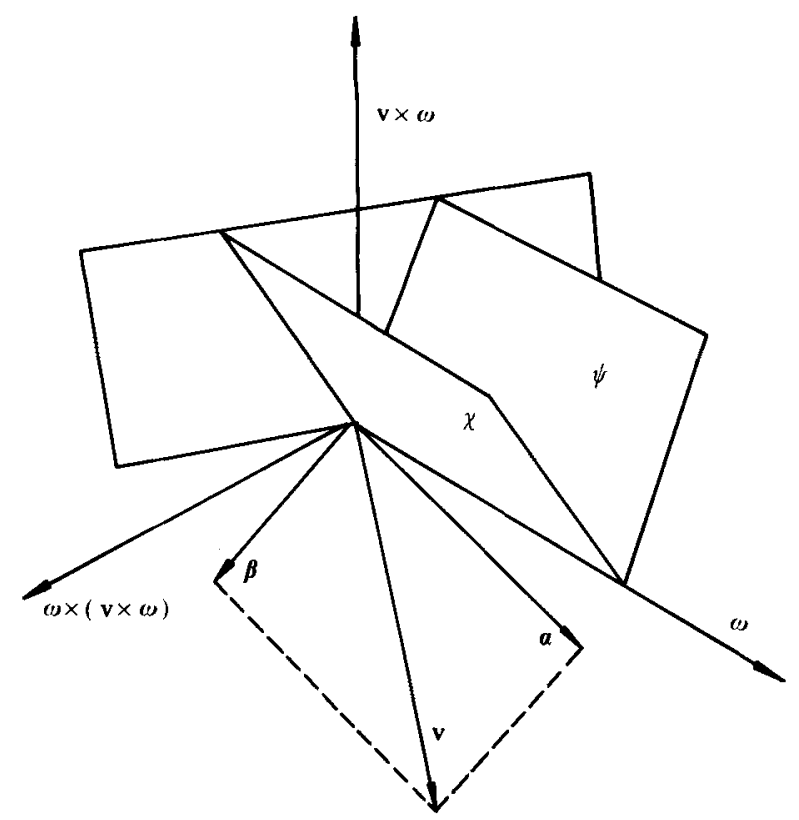

FIgure 1. Potential/complex-lamellar decomposition and Monge's potentials. The vortical component $\beta$ is in the plane perpendicular to $\omega$ and is normal to $\chi$. The potential component $\alpha$ is normal to $\phi$. The $\mathcal{X}$ - and $\psi$-surfaces contain $\omega$.

The general mathematics and characteristics of the complex-lamellar decomposition will be discussed in this section. Consider again a decomposition where the first component is irrotational and the second component gives the vorticity:

$$
\mathbf{v}=\alpha+\beta, \quad \alpha=\nabla \phi, \quad \omega=\nabla \times \beta .
$$

The vortical component $\beta$ is next restricted to be in the plane perpendicular to the vorticity:

$$
\beta .(\nabla \times \beta)=\beta . \omega=0 \text {. }
$$

Kelvin (1851; see Truesdell 1954, p. 23) termed such a vector 'complex lamellar' (potential flows were called lamellar). If and only if a vector is complex lamellar (Phillips 1933, p. 103), it may be represented by two scalar functions: $\beta=\psi \nabla \chi$. From another point of view, if a vector is complex lamellar, there is a function $\psi$ such that $\beta / \psi$ has a potential. The velocity may then be represented by the scalar functions $\phi, \psi$ and $\chi$, which are called Monge's potentials:

$$
\mathbf{v}=\nabla \phi+\psi \nabla \chi, \quad \omega=\nabla \psi \times \nabla \chi
$$

Monge's potentials originated in the study of systems of differential equations when the condition of integrability was not satisfied. Clebsch (1858) employed the Monge potentials in formulating the inviscid fluid-mechanics equations as a variational problem. Lamb (1945, p. 248), Truesdell (1954, p. 27) and Aris (1962, p. 72) give brief discussions of the decomposition of vector fields using Monge's potentials.

Some of the important properties of this decomposition are sketched in figure 1. Surfaces $\psi=$ constant and $\chi=$ constant intersect to define vortex lines. Consider the identities

and

$$
\left.\begin{array}{rl}
\nabla \psi \cdot \omega & =\nabla \psi \cdot \nabla \psi \times \nabla \chi=0 \\
\nabla \chi \cdot \omega & =\nabla \chi \cdot \nabla \psi \times \nabla \chi=0 .
\end{array}\right\}
$$


The necessary and sufficient condition for a vector surface is that the surface normal $(\nabla \psi$ or $\nabla \chi)$ be everywhere perpendicular to the vector. Hence $\psi=$ constant and $\chi=$ constant are equations for vector surfaces of the vorticity. From the physical viewpoint, one of the attractive aspects of this decomposition is that $\psi=$ constant and $\chi=$ constant intersections identify the vortex lines. From its definition (3.1), $\beta$ must lie in the plane which is perpendicular to the vorticity. The potential component $\alpha$ connects $\beta$ to $\mathbf{v}$, and the $\phi$-surface is then perpendicular to $\alpha$. Another consequence of the fact that $\beta$ is perpendicular to $\omega$ is that the projections of $\alpha$ and $v$ onto the vorticity vector are the same: $\mathbf{v} . \omega=\alpha . \omega$.

Monge's potentials are independent wherever the Jacobian is non-zero. The Jacobian turns out to be

$$
\begin{aligned}
J & =\partial(\phi, \psi, \chi) / \partial\left(x_{1}, x_{2}, x_{3}\right)=\nabla \phi .(\nabla \psi \times \nabla \chi) \\
& =\boldsymbol{\alpha} \cdot \boldsymbol{\omega}=\mathbf{v} \cdot \boldsymbol{\omega} .
\end{aligned}
$$

Thus it may become zero because either $\mathbf{v}$ or $\omega$ is zero, i.e. at stagnation points of $\mathbf{v}$ or $\omega$, or because they are perpendicular. Two-dimensional flows are well-known examples where $\mathbf{v}$ and $\omega$ are perpendicular. In these cases, only two potentials are independent, in agreement with the fact that the velocity has only two components. Conversely, the velocity and vorticity are not usually perpendicular in a threedimensional flow. Then $\mathbf{v} \cdot \boldsymbol{\omega}=\mathbf{v} . \nabla \times \mathbf{v}$ is non-zero. That is, $\mathbf{v}$ is not itself complex lamellar nor is it irrotational; therefore both $\alpha$ and $\beta$ components must exist. The vortical component $\beta$ will not be parallel to the velocity because it must be perpendicular to the vorticity.

One significant difference between the present $\alpha, \beta$ decomposition and the common Helmholtz decomposition is that the divergences of $\alpha$ and $\beta$ are not zero. In incompressible flow, the continuity equation $\nabla . v=0$ requires only that the sources of $\alpha$ be the sinks of $\beta$ :

$$
\Delta=\nabla \cdot \boldsymbol{\alpha}=-\nabla \cdot \boldsymbol{\beta}
$$

If the flow is viewed as the superposition of a fluid with potential flow and one with vortical flow, then the present decomposition allows for processes which generate one fluid at the expense of the other.

This completes the characteristics common to all complex-lamellar decompositions. However (3.1) does not completely specify $\alpha$ and $\beta$. There are still an infinite number of ways to decompose a vector field into potential and complex-lamellar components. The first step in selecting a specific decomposition is to choose a direction for $\beta$, the choice being restricted to the plane perpendicular to $\omega$ of course. Since a surface in space is completely specified by its normal vector, choosing the $\beta$-direction is equivalent to choosing a set of surfaces $\chi=$ constant. Choosing a direction for $\beta$ or choosing a set of $\chi$-surfaces amounts to the same thing, and determines the vector decomposition. To show that this is indeed the case, one considers the vector field $\mathbf{v}$ to be given, assumes the vortex surfaces $\chi=$ constant and proceeds in principle to compute $\phi$ and $\psi$. This process will be considered next.

For a given vector field the problem of determining the Monge potentials is called Pfaff's problem (Brand 1947, pp. 227-230; Ince 1956, p. 57). Two methods of solution will be reviewed. In both methods, the first step is to choose a set of $\chi$-surfaces (vector surfaces of $\omega$ ). When $\omega \neq 0$, there are two independent choices for these 
surfaces; any other set of vector surfaces can be represented as a function of the original choices. In the first method, any curve in the $\mathcal{X}$-surface is chosen and the projection of $\mathbf{v}$ onto the curve is formed. Then from (3.1)

$$
\begin{aligned}
\mathbf{v} . d \mathbf{x} & =\nabla \phi . d \mathbf{x}+\psi \nabla \chi . d \mathbf{x}=d \phi+\psi d \chi \\
& =d \phi \quad \text { on } \quad \chi=\text { constant. }
\end{aligned}
$$

Therefore the potential $\phi$ is given by

$$
\phi-\phi_{0}(\chi)=\int_{x_{0}}^{x} \mathbf{v} \cdot d \mathbf{x} \quad \text { on } \quad \chi=\text { constant }
$$

With $\chi$ and $\phi$ known, back substitution into (3.2) yields $\psi$ from the equation

$$
\psi \nabla \chi=\mathbf{v}-\nabla \phi
$$

The reference value $\phi_{0}(\gamma)$ changes from one $\chi$-surface to the next.

The second method determines $\psi$ first, then deduces $\phi$ from (3.2). Again, a set of $x$-surfaces is chosen. The vorticity field may be deduced from the known velocity field, and the vector product of $\omega$ and a differential line element produces the equation

$$
\omega \times d \mathbf{x}=\nabla \chi d \psi-\nabla \psi d \chi
$$

When $d \mathbf{x}$ lies in the $\chi$-surface, the vector $\omega \times d \mathbf{x}$ is perpendicular to that surface. Take any unit normal $\mathbf{n}$ which does not lie in the $\chi$-surface and form the inner product

$$
\begin{aligned}
\mathbf{n} . \boldsymbol{\omega} \times d \mathbf{x} & =\mathbf{n} \times \boldsymbol{\omega} . d \mathbf{x}=\mathbf{n} . \nabla \chi d \psi-\mathbf{n} . \nabla \psi d \chi \\
& =\mathbf{n} . \nabla \chi d \psi \quad \text { on } \quad \chi=\text { constant }
\end{aligned}
$$

Interchanging the order of operations and solving gives the expression for $\psi$ :

$$
\psi-\psi_{0}(\chi)=\int_{x_{0}}^{x} \frac{\mathbf{n} \times \boldsymbol{\omega} \cdot d \mathbf{x}}{\mathbf{n} \cdot \nabla \chi} \text { on } \chi=\text { constant. }
$$

Now with $\chi$ and $\psi$ known, the potential $\phi$ is found by integrating along any path the relationship

$$
d \phi=\nabla \phi \cdot d \mathbf{x}=(\mathbf{v}-\psi \nabla \chi) \cdot d \mathbf{x}
$$

The choice of $\chi$-surfaces would simplify the integration as in (3.7).

One of the important physical characteristics of the decomposition is illustrated by (3.11). The integrand of (3.11) is non-zero only when the vorticity is non-zero. In a region of the flow which is purely potential, $\psi$ and $\beta$ are zero. The vortical component $\beta$ will exist only in regions where vorticity exists to make a contribution to the integrand of (3.11).

The decomposition was begun by choosing a set of $\chi$-surfaces. The second step is to fix a numbering system for the $\chi$-surfaces. The specific numbering system does not affect the vector components. This may be proved by considering a renumbering of the surfaces $\hat{\chi}=g(\chi)$. The surfaces $\hat{\chi}=$ constant and $\chi=$ constant are the same but have different numbers. Then $\nabla \hat{\chi}=g^{\prime} \nabla \chi$ and substitution into (3.11) shows that $\hat{\psi}=\left(1 / g^{\prime}\right) \psi$, therefore one may conclude that the vector $\beta=\hat{\psi} \nabla \hat{\chi}=\psi \nabla \chi$ is unchanged. Although the $\chi$-surfaces were only renumbered, the new $\hat{\psi}$-surfaces are 


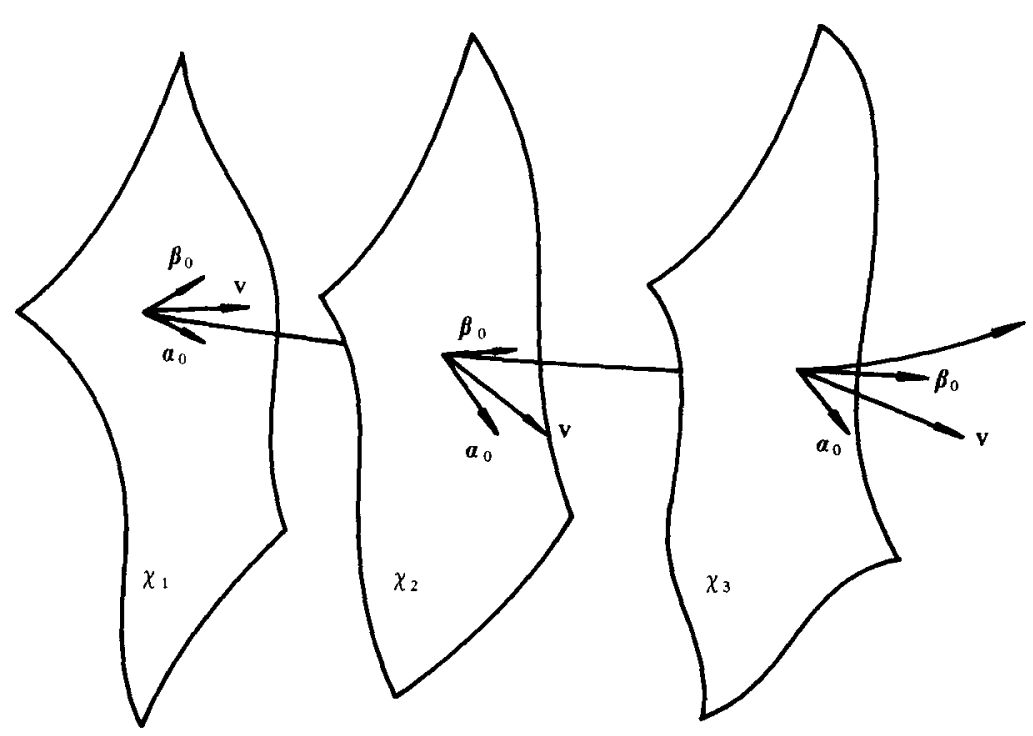

FIGURE 2. Reference values of $\beta_{0}$ are prescribed on any line which connects all $\chi$-surfaces. This determines the reference values $\psi_{0}(\chi)$ and $\phi_{0}(\chi)$.

not the same as the old $\psi$-surfaces. Also note that the numbering system may change with time.

The third and final step is to fix the reference values $\phi_{0}$ and $\psi_{0}$. This must be done for each $\chi$-surface. It amounts to choosing either $\alpha$ or $\beta$ on a line connecting all the $\chi$-surfaces. Consider a line connecting the $\chi$-surfaces as shown in figure 2 . Along this line one chooses the magnitude of $\beta_{0}$ (the direction is already fixed by $\left.(\nabla \chi)_{0}\right)$. Then $\psi_{0}$ values are found from $\beta_{0}=\psi_{0}(\nabla \chi)_{0}$. Since $\alpha_{0}-\beta_{0}$ is known, the reference values $\phi_{0}$ are found using $\alpha_{0}=\nabla \phi$. Integrating along the line yields the equation

$$
\phi_{0}-\phi_{00}=\int \alpha_{0} . d \mathbf{x} \text { on the line across the } \chi \text {-surface. }
$$

The integration constant $\phi_{00}$ is the value of $\phi_{0}$ on one particular $\mathcal{X}$-surface. The value of $\phi_{00}$ does not affect $\alpha=\nabla \phi$, nor does it affect the values of $\psi$ and $\chi$. We have now in principle made a unique decomposition of a given velocity field.

The second important physical characteristic of the complex-lamellar decomposition is that it allows one to trace the history of vortex lines in a viscous fluid. Vortex lines are marked as the intersection of $\psi$ - and $\chi$-surfaces. Thus two numbers $\psi$ and $\chi$ identify a vortex line and allow its time history to be traced. A unique, completely specified decomposition implies specific motion for the vortex lines. On the other hand, if the vector decomposition is changed by choosing a different $\beta$-direction (different $\chi$-surfaces), the vortex-line history may change. Even with the same vector decomposition, i.e. the same $\alpha$ and $\beta$, the vortex-line history can be modified by changing the $\mathcal{X}$-surface numbering system.

The fact that there are many ways to follow vortex lines is not at odds with Helmholtz's theorem that vortex lines follow the fluid particles in an inviscid fluid. The $\psi$-and $\mathcal{X}$-surfaces may be so chosen that a particular intersection follows the fluid, 
but they may also be chosen in other ways. For example, in a steady flow the $\psi$ and $\mathcal{X}$-surfaces would usually be chosen to give stationary vortex lines.

A unique complex-lamellar decomposition specifies the time development of vortex lines. The freedom to make the decomposition in many ways provides an opportunity to define vortex lines in a manner that has dynamic significance.

Further insight into the complex-lamellar decomposition is gained by relating it to the Helmholtz decomposition. One can in principle solve for the potential flow $\alpha=\nabla \phi$. The governing equation is

$$
\nabla . \alpha=\nabla \cdot \mathbf{v}-\nabla \cdot \beta, \quad \nabla^{2} \phi=\tilde{\Delta}+\Delta,
$$

where the sources $\bar{\nabla} \equiv \nabla . \mathrm{v}$ and $\Delta=-\nabla . \beta$ are assumed known. The solution, including the harmonic function, is

$$
\begin{aligned}
\alpha & =\nabla \Phi+\frac{1}{4 \pi} \int \frac{\mathbf{r}\left(\Delta^{\prime}+\Delta^{\prime}\right)}{|\mathbf{r}|^{3}} d V^{\prime} \\
& =\tilde{\alpha}+\frac{1}{4 \pi} \int \frac{\mathbf{r} \Delta^{\prime}}{|\mathbf{r}|^{3}} d V^{\prime} .
\end{aligned}
$$

The vortical component is found from $\alpha+\beta=\tilde{\alpha}+\tilde{\beta}$ as

$$
\beta=\tilde{\beta}-\frac{1}{4 \pi} \int \frac{\mathbf{r} \Delta^{\prime}}{|\mathbf{r}|^{3}} d V^{\prime} .
$$

This representation of $\alpha$ and $\beta$ is valid if they are bounded. If they become unbounded, then a singularity, such as a point source, must be added to the right-hand sides. Since $\mathbf{v}$ is bounded, the same singularity occurs in both $\alpha$ and $\beta$, but with opposite signs. In Helmholtz's decomposition $\tilde{\beta}$ exists at remote positions where the vorticity is zero. In the complex-lamellar decomposition $\beta$ is zero where the vorticity is zero. Thus (3.15) implies that a distribution of sources $\Delta$ within the vortical region induces a flow which exactly cancels $\tilde{\beta}$ at remote positions. The flexibility in the $\alpha, \beta$ decomposition reflects the fact that there are many ways to choose a source distribution within a region which produces a given harmonic potential outside that region (Kellogg 1953, p. 197).

\section{Dynamic equations}

A specific choice of $\mathcal{X}$-surfaces, or equivalently the $\beta$-direction, is needed to complete the decomposition. Turbulence is only one of several types of flows in which there is strong interaction between vortical and potential components. One can make different choices of $\beta$ depending upon the physical situation. In fact, in principle we could make different definitions for wall turbulence and free shear layers. In this section, the dynamic equations are examined to see how the final choice of $\beta$-direction influences various terms. The object is to specify the $\beta$-direction in a way which has dynamic significance.

The momentum equation may be written in a form which splits the convective terms $\mathbf{v} \cdot \nabla \mathbf{v}=\nabla\left(\frac{1}{2} v^{2}\right)-\mathbf{v} \times \boldsymbol{\omega}$ into a pressure-like component and the transport of momentum perpendicular to the vortex lines. The term $\mathbf{v} \times \boldsymbol{\omega}$ has been called the vortex force and also the Lamb vector. It is the $v \times \omega$ component which leads to the Reynolds-stress terms in turbulence (Tennekes \& Lumley 1972, p. 78). Substituting the decomposition into the $\mathbf{v} \times \boldsymbol{\omega}$ terms, the momentum equation reads

$$
\partial \mathbf{v} / \partial t+\nabla\left(p / \rho+\frac{1}{2} v^{2}\right)=\alpha \times \omega+\beta \times \omega+\nu \nabla \times \omega
$$




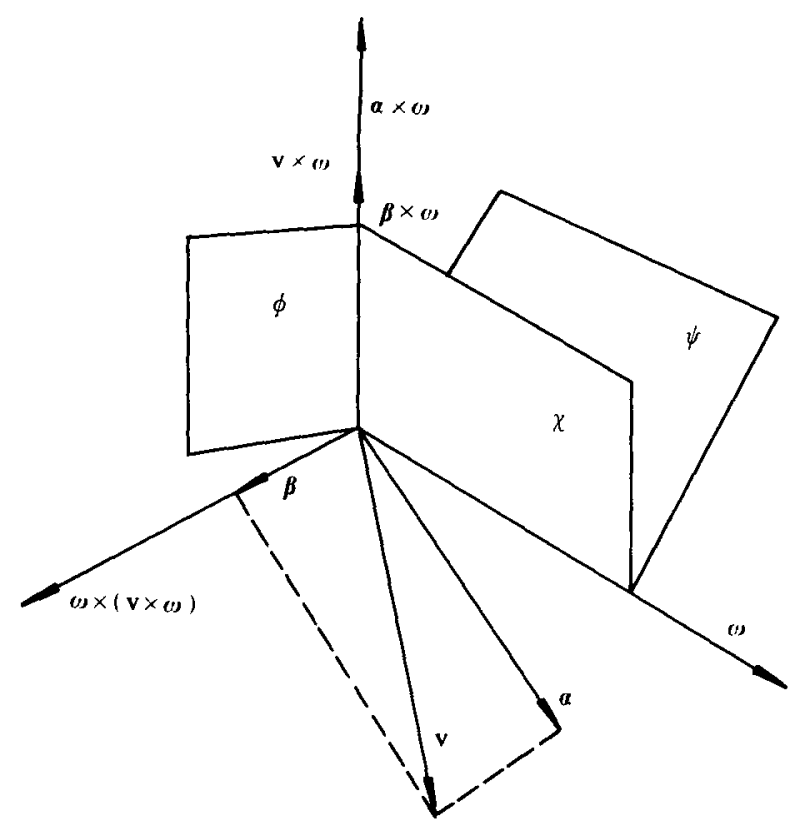

Figure 3. Decomposition with $\beta$-direction chosen in $\mathbf{v}, \omega$ plane. Vortex force $\mathbf{v} \times \omega, \beta \times \omega$ and $\alpha \times \omega$ collinear. The $\phi$ - and $\chi$-surfaces contain $\mathbf{v} \times \omega$, while the $\psi$-and $\chi$-surfaces contain $\omega$.

For non-zero $\alpha$ and $\beta, \alpha \times \omega$ could only be eliminated by having $\alpha$ and $\omega$ parallel. This is too restrictive, since it would determine both the direction and the magnitude of $\beta$ (see figure 1). Therefore it is not possible to choose the $\beta$-direction such that all of the Reynolds stress is attributed to one velocity component. A physical interpretation is that the vortex force $\mathbf{v} \times \boldsymbol{\omega}$ is concerned only with the velocity component perpendicular to the vortex lines. Whether this velocity arises from potential or vortical motions is immaterial. This is an essential interaction between the potential and the vortical flows.

Since one cannot eliminate either $\alpha \times \omega$ or $\beta \times \omega$, the next best possibility would be to arrange things such that they are in the same direction and will simply add in magnitude to provide $\mathbf{v} \times \boldsymbol{\omega}$. This is accomplished by choosing the $\beta$-direction to be in the $\mathbf{v}, \omega$ plane in addition to the requirement that $\beta$ is perpendicular to the vorticity (see figure 3). The $\chi$-surfaces now take on added physical significance. They are vector surfaces of the vortex force or Lamb vector $\mathbf{v} \times \boldsymbol{\omega}$, as well as vector surfaces of the vorticity. Each $\mathcal{X}$-surface contains an orthogonal set of lines: the vortex lines and the vortex-force or Lamb lines. Intersection of a $\psi$-surface with the $\chi$-surface gives a vortex line, while intersection of a $\phi$-surface with the $\chi$-surface gives a vortex-force line.

As an aside, note that (4.1) can be integrated along vortex lines for a flow where viscous diffusion may be neglected. The motion in the outer turbulent region is thought to be of this type. The term $\mathbf{v} \times \boldsymbol{\omega}$ is perpendicular to the vortex lines and will not make any contribution to the integral. The unsteady term is written as

$$
\frac{\partial \mathbf{v}}{\partial t}=\nabla\left[\frac{\partial \phi}{\partial t}+\psi \frac{\partial \chi}{\partial t}\right]-\frac{\partial \chi}{\partial t} \nabla \psi+\frac{\partial \phi}{\partial t} \nabla \chi
$$

The last two terms vanish when integrated along a vortex line since $\omega \cdot \nabla \psi=\omega \cdot \nabla \chi=0$. 
The final equation which holds along a vortex line is

$$
\frac{\partial \phi}{\partial t}+\psi \frac{\partial \chi}{\partial t}+\frac{p}{\rho}+\frac{1}{2} v^{2}=C(t)
$$

If the flow has no vorticity $\psi=0$, and the more familiar form of the unsteady Bernoulli equation is retrieved (Lamb 1945, p. 249).

Next the vorticity transport equation will be considered. Fluctuations in vorticity are one of the accepted characteristics of turbulent flows. The vorticity equation is

$$
D \boldsymbol{\omega} / D t=\boldsymbol{\omega} \cdot \nabla \mathbf{v}+\nu \nabla^{2} \boldsymbol{\omega}=\boldsymbol{\omega} \cdot \nabla \boldsymbol{\alpha}+\boldsymbol{\omega} \cdot \nabla \beta+\nu \nabla^{2} \boldsymbol{\omega} .
$$

A term of major interest is the production of vorticity by turning and stretching. It was pointed out previously that the strain rate cannot be isolated into one component. Therefore the $\beta$-direction cannot be chosen such that $\nabla \beta$ has any special symmetries. Likewise, the convective terms $\alpha . \nabla \omega$ and $\beta$. $\nabla \omega$ cannot be attributed to only one component. There are no obvious simplifications possible for this equation.

There is one dynamic equation that simplifies when $\beta$ is chosen in the $\mathbf{v}, \omega$ plane. Forming the scalar product of the momentum equation with $\alpha$ then with $\beta$, and noting that $\boldsymbol{\alpha} . \mathbf{v} \times \boldsymbol{\omega}=0$ and $\boldsymbol{\beta} . \mathbf{v} \times \boldsymbol{\omega}=0$ yields two independent equations:

and

$$
\left.\begin{array}{l}
\alpha . \partial \mathbf{v} / \partial t+\alpha \cdot \nabla\left(\frac{1}{2} v^{2}\right)=-\rho^{-1} \boldsymbol{\alpha} . \nabla p+\nu \alpha . \nabla \times \omega \\
\beta . \partial \mathbf{v} / \partial t+\beta . \nabla\left(\frac{1}{2} v^{2}\right)=-\rho^{-1} \beta . \nabla p+\nu \beta . \nabla \times \omega .
\end{array}\right\}
$$

The sum of these equations is the usual kinetic energy equation. The specific choice of the $\beta$-direction, such that $\beta, v$ and $\omega$ are coplanar, allows the kinetic energy equation to be split into two symmetric and independent equations. This may be useful, as the kinetic energy equation is frequently used in turbulence modelling.

An essential element of a three-dimensional flow is that the vortex lines and the streamlines do not meet at right angles. A measure of the three-dimensionality is given by v.w. A dynamic equation for $\mathbf{v} . \omega$ can be derived by dotting $\mathbf{v}$ with the vorticity equation, dotting $\omega$ with the momentum equation, then rearranging and adding the equations to produce

$$
D(\mathbf{v} \cdot \boldsymbol{\omega}) / D t=-\rho^{-1} \boldsymbol{\omega} \cdot \nabla p+(\mathbf{v} \boldsymbol{\omega}): \mathbf{S}+\nu\left[\boldsymbol{\omega} \cdot \nabla^{2} \mathbf{v}+\mathbf{v} \cdot \nabla^{2} \boldsymbol{\omega}\right] .
$$

The inner product $\mathbf{v} . \omega$ may be an interesting property of a flow, however it changes because of changes in the magnitudes of $\mathbf{v}$ and $\omega$, as well as changes in the angle between them. Letting $\gamma$ be the cosine of this angle gives $\mathbf{v} . \omega=v \omega \gamma$, where $v$ and $\omega$ are the magnitudes of the vectors. Multiplying (4.6) by $\mathbf{v} . \omega$ produces an equation for the substantial derivative of $\frac{1}{2} v^{2} \omega^{2} \gamma^{2}$. After splitting this derivative, $D \omega^{2} / D t$ and $D v^{2} / D t$ can be eliminated by using the dynamic equations for these quantities. Some slight rearrangement yields the final equation for the cosine of the angle between $\mathbf{v}$ and $\omega$ :

$$
\frac{D \gamma}{D t}=-\frac{1}{\rho v} \mathbf{W} \cdot \nabla p+\left(\mathbf{V t}_{\omega}\right): \mathbf{S}+\nu\left[\frac{1}{v} \mathbf{W} \cdot \nabla^{2} \mathbf{v}+\frac{1}{\omega} \mathbf{V} \cdot \nabla^{2} \boldsymbol{\omega}\right]
$$

where $\mathbf{W}=\mathbf{t}_{\omega}-\gamma \mathbf{t}_{v}, \mathbf{V}=\mathbf{t}_{v}-\gamma \mathbf{t}_{\omega}$ and $\mathbf{t}_{\omega}$ and $\mathbf{t}_{v}$ are unit vectors tangential to the vorticity and velocity vectors. The special directions $\mathbf{W}$ and $\mathbf{V}$ play a prominent role in changing the angle between $\mathbf{v}$ and $\boldsymbol{\omega}$. In a two-dimensional flow, or a nearly twodimensional flow such as the sublayer, the angle between $\mathbf{v}$ and $\omega$ is $90^{\circ}$, so that $\mathbf{W}=\mathbf{t}_{\omega}$ and $\mathbf{V}=\mathbf{t}_{\boldsymbol{v}}$. This is a point of reference in interpreting the terms in (4.7). 
The first term in (4.7) shows that the pressure gradient inducing flow in the W direction turns the velocity vector into the vorticity vector, thereby closing the angle $\gamma$. It is scaled by $1 / v$ because a given pressure gradient will cause a larger rate of closure if $v$ is small than if it is large. This is also the only term of a non-local nature. The pressure field depends upon the surrounding flow, while all other terms contain only the local velocity and its derivatives. The first term illustrates how a pressure gradient imposed in the vorticity direction of a two-dimensional flow causes the flow to become three-dimensional. The next term $\mathbf{V t}_{\omega}: \mathbf{S}$ represents the closure of the angle because of the local strain rate $\mathbf{S}$. The last two terms represent viscous diffusion into the point in question; $W . \nabla^{2} v$ represents diffusion of momentum which is aligned with $W$ and $V . \nabla^{2} \omega$ represents diffusion of vorticity which is aligned with the $V$ direction.

When the $\beta$-direction is chosen in the $\mathbf{v}, \omega$ plane, the relation between the potential/ complex-lamellar decomposition and the $\gamma$ equation is very simple. Since $\beta$ and $\omega$ are at right angles, $\gamma$ may be reinterpreted as the sign of the angle between the velocity and the vortical component $\beta$. Other choices of the $\beta$-direction will not give a simple relationship.

After examining the several dynamic equations, it seems that choosing $\beta$ to be in the $\mathbf{v}, \boldsymbol{\omega}$ plane is best. This choice breaks the vortex force $\mathbf{v} \times \boldsymbol{\omega}$ into two collinear parts $\boldsymbol{\alpha} \times \boldsymbol{\omega}$ and $\boldsymbol{\beta} \times \boldsymbol{\omega}$. Also, with this choice the Monge potentials have additional physical meaning. The $\phi$ - and $\chi$-surfaces are vector surfaces of the vortex-force vector $\mathbf{v} \times \omega$ and their intersections give lines tangential to $\mathbf{v} \times \boldsymbol{\omega}$. Choosing $\beta$ to be in the $\mathbf{v}, \boldsymbol{\omega}$ plane also allows the kinetic energy equation to be split into two independent equations.

\section{Decomposition of wall turbulence}

Application of the complex-lamellar decomposition to an incompressible turbulent wall layer will be described in this section. The $\beta$-direction will be chosen to be in the $\mathbf{v}, \boldsymbol{\omega}$ plane as discussed previously. In a purely two-dimensional flow, where $\mathbf{v}$ and $\boldsymbol{\omega}$ are perpendicular, this means that $\beta$ is in the same direction as $\mathbf{v}$ and that the $\chi$ surfaces lie across the layer. The turbulent case is of course much more complicated, and the best one can do is discuss how this assumption leads to well-defined $\beta$-directions throughout the region. The interface, the interior and the wall regions will be considered in turn.

The interface between turbulent and non-turbulent flow is defined as the surface where the vorticity vanishes in some sense. Since $\nabla . \omega=0$ in addition to $\omega=0$, the same requirements are imposed on the vorticity at the interface as are encountered for the velocity at a solid wall. By arguments similar to those for defining a limiting streamline on a wall, the limiting vortex lines in the interface may be defined (one can also show that $\partial \omega_{n} / \partial n=0$ ). The fact that $\omega$ has a limiting direction in the interface is important, since the $\mathbf{v}, \boldsymbol{\omega}$ plane and the $\boldsymbol{\beta}$-direction are therefore well defined (see figure 4). This means that $\nabla \chi$ is not zero at the interface. Now at the interface $\beta=\psi \nabla \chi=0$, so it must follow that the in terface is a surface $\psi=0$. Furthermore since the vorticity $\omega=\nabla \psi \times \nabla \chi$ is zero, the gradient $\nabla \psi$ should also be zero at the interface. These are idealizations. There must be a slight amount of vorticity in the non-turbulent region so the $\chi$-surfaces actually cover that region also. Assigning $\psi=0$ in the non-turbulent region gives that region its completely potential character with $\mathbf{v}=\boldsymbol{\alpha}$. 


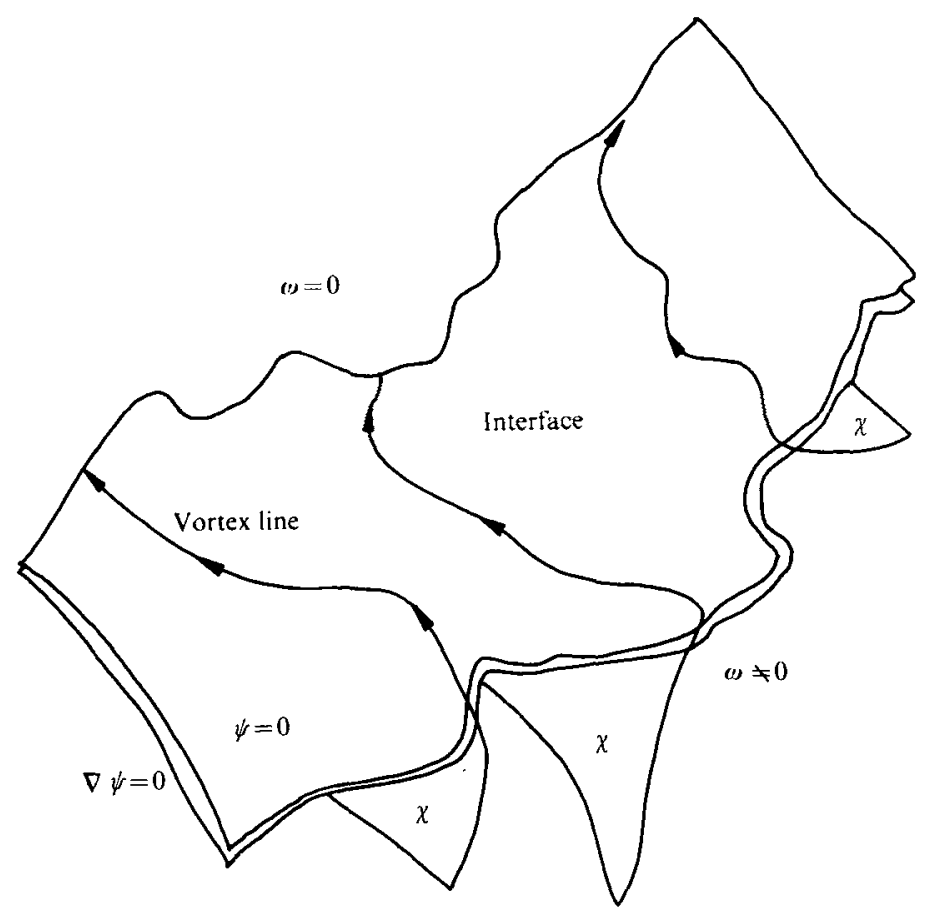

Figure 4. Turbulent/non-turbulent interface. $\psi=0, \nabla \psi=0$, $\chi$-surfaces contain surface vortex lines.

The flow within the turbulent region is discussed next. The $\beta$-direction (and $\nabla \chi$ ) is defined by the velocity and vorticity vectors. If one of these is zero then $\nabla \chi$ may not be defined. In an attached flow the velocity is never zero away from the wall. Therefore $\beta$ and $\nabla \chi$ have a well-defined direction everywhere in the turbulent region, with the possible exception of places where $\omega=0$. It is not necessary that $\nabla \chi$ is singular at these points, but it is possible. We have just discussed the interface where $\omega=0$ on a surface, but $\nabla \chi$ was still well defined.

It is possible that the vorticity is zero at local points within the turbulent region. The vorticity obeys a convective diffusive differential equation. It might be expected, especially in light of the fact that vortex tubes have a constant strength, that all three components of the vorticity will not be zero simultaneously. However, the vorticity equation

$$
D \omega / D t=\omega \cdot \nabla \mathbf{v}+\nu \nabla^{2} \boldsymbol{\omega}
$$

differs from the heat-conduction equation by the vortex turning and stretching term. This term may in some way produce a local zero in the vorticity. Experimental observations tend to support this view. Aircraft trailing vortices, for example, have been observed to loop and pinch off a ring vortex. The pinching-off process, as depicted in figure $5(a)$, must have two vortex lines at one point, and therefore a vorticity stagnation point. These considerations lead to the assumption that there may be points within the turbulent region where the vorticity vector is zero, but that these points are isolated. If a singularity in $\nabla \chi$ and $\beta$ occurs, it must be matched by a corresponding singularity in $\alpha$ since $\mathbf{v}$ is bounded. Thus the singularity will be a point potential singularity. 


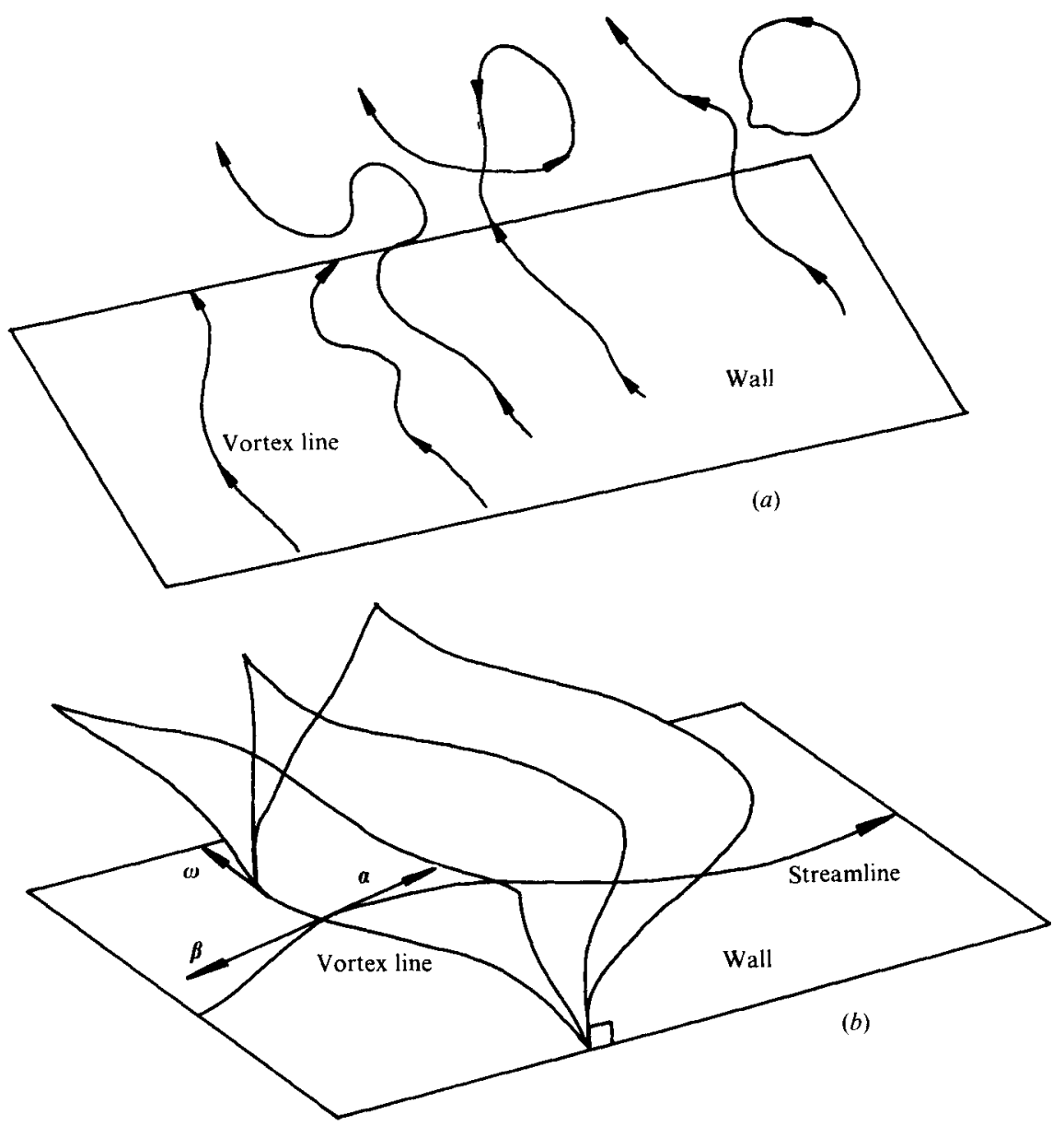

FIGURE 5. Vortex lines: $(a)$ pinching-off process with internal 'stagnation' point $\omega=0$; $(b)$ vortex lines in solid wall. $\psi$-, $\chi$ - and $\phi$-surfaces contain vortex lines, $\chi$ - and $\phi$-surfaces are perpendicular to the wall and $\beta=-\alpha$ is in the direction of the streamline.

Finally, the situation at the wall is considered. The direction of the velocity is given by the limiting streamlines. The vortex lines (for a Newtonian fluid) lie in the wall and are perpendicular to the streamlines. Choosing the $\beta$-direction in the $v, \omega$ plane means that $\beta$ is directed along the wall in the streamline direction. Consequently, the $\mathcal{X}$-surfaces are perpendicular to the wall and their intersections with the wall form the vortex lines as shown in figure $5(b)$. The no-slip condition requires $\beta=-\alpha=-\nabla \phi$, so $\alpha$ also lies in the wall, with $\phi$-surfaces which are tangential to the $\chi$-surfaces. Vortex lines are given by the intersections of $\chi$-and $\psi$-surfaces; hence the $\psi$-surfaces also intersect the wall on the vortex lines but not at right angles. Another possibility is that the wall is the surface $\psi=1$. This occurs only for a special choice of the $\chi$-surface numbering system. Two final facts are noted. From the relation between the wall shear stress and vorticity $(\boldsymbol{\tau}=\mu \boldsymbol{\omega} \times \mathbf{n})$ one may show that $\partial \boldsymbol{\beta} / \partial n=\partial \mathbf{v} / \partial n$. This also requires that $\partial \alpha / \partial n=0$ at the wall.

The major points of interest so far in this section are the $\chi$-surfaces. They must leave the wall at $90^{\circ}$, heading into the turbulent region. In the turbulent region $\nabla \chi$ 


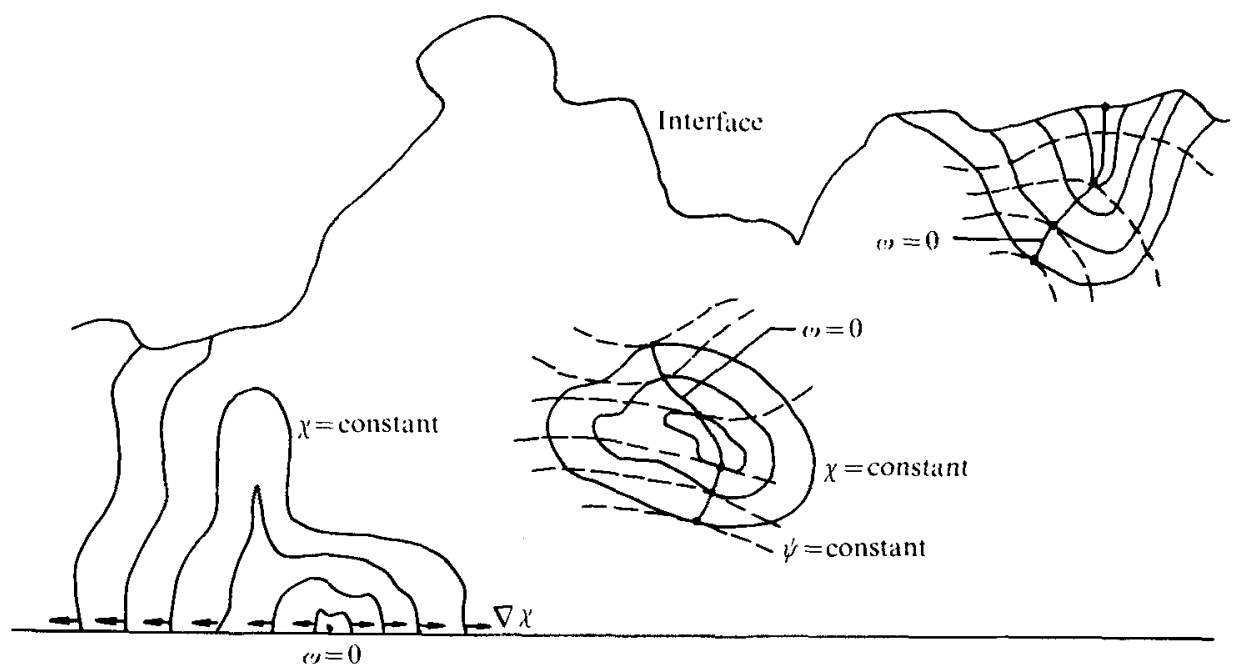

Figure 6. Configurations of $\chi$-surfaces which give lines where $\omega=0$.

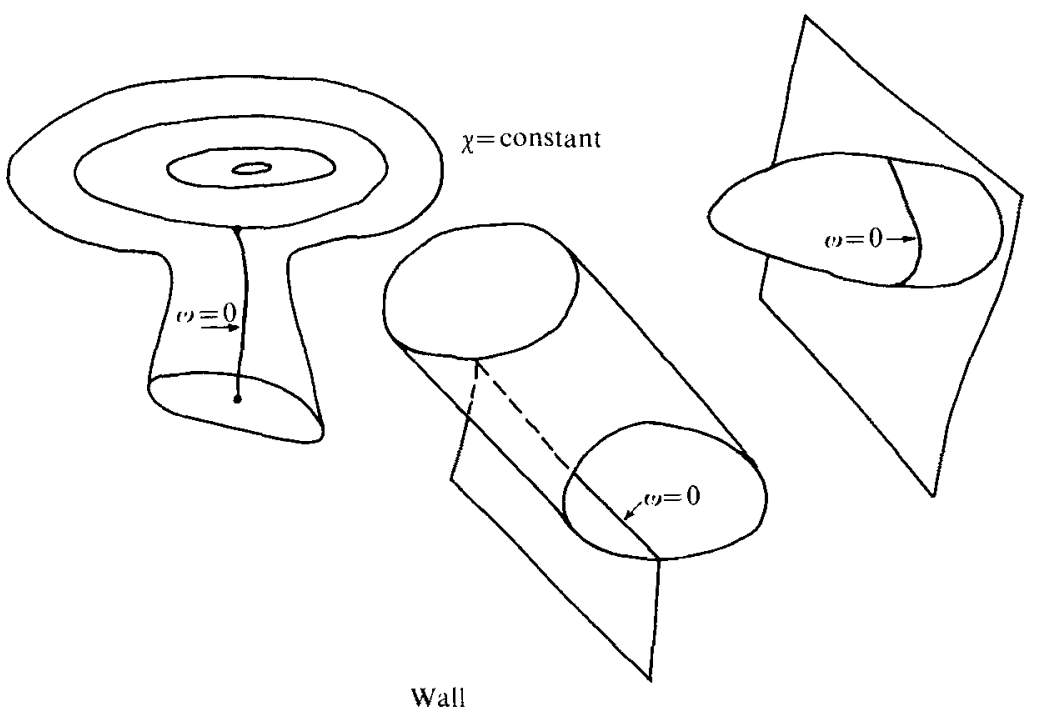

Figure 7. Configurations of $\chi$-surfaces which give $\omega=0$ on a line.

is well defined, with the possible exception of isolated points where the vorticity is zero. The $\chi$-surfaces arrive at the turbulent/non-turbulent interface at some angle, and the interface is the surface $\psi=0$.

A complete complex-lamellar decomposition requires one to specify a set of $\chi$ surfaces, reference values of $\beta_{0}$ on a line connecting the $\chi$-surfaces, and the $\chi$-surface numbering system. The reference values for the turbulent wall layer are taken at the interface where $\beta_{0}=0$. The next question is, does this supply reference values for all the $\chi$-surfaces? Are there any $\mathcal{X}$-surfaces which do not ultimately intersect the interface? The answer is that all $\chi$-surfaces do intersect the interface if some fairly plausible assumptions are made. In the next few paragraphs several different configurations 
of $\chi$-surfaces which do not intersect the interface will be discussed. Each of these will be shown to violate an assumption about the flow.

Figures 6 and 7 show several configurations of $\chi$-surfaces which are not connected to the interface. Consider the possibility that the $\chi$-surfaces which leave the wall return at some downstream point. If this happens, there are two wall vortex lines which have the same value of $\chi$. Therefore $\chi$ must have a maximum or minimum value on an intermediate wall vortex line. Since the $\chi$-surfaces are perpendicular to the wall, this means that $\nabla \chi=0$ on that vortex line. This is prohibited, since the vorticity $\boldsymbol{\omega}=\nabla \psi \times \nabla \chi$ would be zero, resulting in zero shear stress and a local line of flow separation. The $\chi$-surfaces, once they leave the wall, never return as long as the flow is attached.

In the interior of the turbulent fluid, one might envisage structures where the $\chi$ surfaces are closed, so that they enclose a region of the fluid. This structure cannot be connected to the wall by a line, since that means that the surrounding $\chi$-surfaces form closed loops on the wall and again produce a violation of the attached-flow assumption. If a $\chi$-surface connects the enclosed region, there is a line of points where $\nabla \chi$ is undefined. Choosing $\beta$ to be in the $\mathbf{v}, \boldsymbol{\omega}$ plane produces a well-defined direction for $\nabla \mathcal{X}$ except when $\boldsymbol{\omega}=0$. The additional assumption that $\boldsymbol{\omega}=\mathbf{0}$ only at isolated points then prohibits this configuration. The interior of a region of closed $\chi$-surfaces also gives invalid results. Consider the $\psi$-surfaces within the region. If they coincide with the $\chi$-surfaces then the vorticity is zero throughout the region, since $\nabla \psi$ and $\nabla \chi$ are parallel. If they do not coincide, then $\psi$ reaches a maximum and a minimum on each $\mathcal{X}$-surface. At these points, the $\chi$ - and $\psi$-surfaces are tangential, and again a prohibited line where $\omega=0$ is obtained.

Another configuration to consider is where the $\mathcal{X}$-surfaces leaving the wall bend over and roughly align themselves with the streamlines, so that they never intersect the interface. If this happens, then $\beta$ is roughly perpendicular to the wall and the vorticity is nearly in the same direction as the velocity. The vortex force $\mathbf{v} \times \boldsymbol{\omega}$, which is responsible for the Reynolds stress, becomes small and generally directed in the spanwise direction. This could occur locally, however we do not anticipate that it could continue indefinitely. In general then, the $\chi$-surfaces proceed across the layer and intersect the interface. At this interface the reference values $\beta_{0}=0$ are assigned and the vector decomposition completed.

\section{Vortex-line history}

At any instant one can in principle draw the vortex lines in a fluid. From one instant to the next we cannot tell which vortex lines have vanished and which have moved to a new position without giving the lines an identity. The complex-lamellar decomposition identifies vortex lines as the intersections of $\psi$ - and $\chi$-surfaces. Two specific values of $\psi$ and $\chi$ mark each line. This section considers the problem of choosing the $\chi$-numbering system and how it should change with time. Specifying the numbering system will determine the vortex-line motion. Consider for a moment the steady flow of an inviscid fluid carrying vorticity. A certain complex-lamellar decomposition is made and the $\chi$-numbering system is chosen to be independent of time. The vortex lines are then stationary. Making the $\chi$-numbering system change with time gives motion to the $\psi, \chi$ intersections. Helmholtz's theorem that vortex lines in an inviscid 


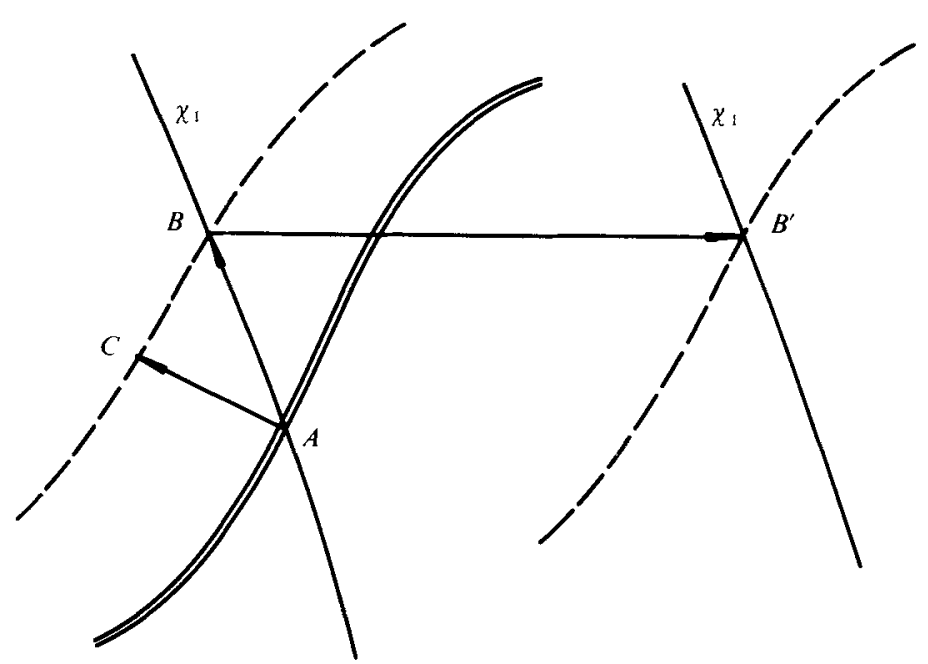

FIGURE 8. Vortex-line motion prescribed by choosing the $\chi$-numbering system at the turbulent/ non-turbulent interface. The interface at time 0 is given by the double line. The solid line is the same $\chi$-surface for both time 0 and time $1 . A$ is the $\chi$-intersection with the interface at time 0 while $B^{\prime}$ is the intersection at time 1. The dashed line is the interface at time 1. $A C$ is the diffusion distance of the interface during the time interval. $B B^{\prime}$ is the distance the material point $B$ has travelled during the interval. The direction of $A C$ is $n_{i}$ and the direction of $A B$ is $\mathbf{t}_{v} \times \mathbf{t}_{\omega}$.

flow are material lines implies a special choice for the time dependence of the $\chi$ numbering system (Lamb 1945, p. 249). This special choice is attractive, since then the motion of the vortex lines is in agreement with the dynamic equation governing the vorticity of an inviscid fluid.

Including the influence of viscosity allows diffusion and destruction of vortex lines. The proper choice of $\mathcal{X}$-surface motion which is compatible with the dynamics is no longer clear. However, in the case of boundary layers a reasonable choice may be made. Vorticity actually contaminates the whole field out to infinity, where the external stream is a pure potential flow. Requiring that the $\chi$-surfaces at infinity travel at the external stream velocity will make the $\psi, \chi$ intersections follow the flow at this point. Thus, at the place where the flow becomes irrotational, the vortex lines will move according to Helmholtz's theorem.

This idea must be modified slightly when the concept of a turbulent/non-turbulent interface is introduced. Figure 8 shows the interface at two different times. Material point $A$ is on the interface initially and moves to $A^{\prime}$ after a time $d t$. The surface $\chi=$ constant which initially contains $A$ continues into the non-turbulent fluid. The direction of the $\chi$-surface is $\mathbf{t}_{v} \times \mathbf{t}_{\omega}$, under the assumption that the $\beta$-direction is in the $\mathbf{v}, \omega$ plane. The material point $B$ on the $\chi$-surface is so located that it is on the interface at time $t+d t$. Then throughout the time interval $B$ is in irrotational motion. If the $\chi$-surface numbering system is chosen such that $B$ is on the same surface throughout the interval $d t$, then the $\chi$-surface will follow the fluid particle, and will be in agreement with Helmholtz's theorem and the dynamic vorticity equation.

The motion of the $\chi$-numbering system can in principle be specified by giving the velocity of a $\mathcal{X}$-surface at the interface, i.e. $\lim _{d t \rightarrow 0} \overline{A B^{\prime}} / d t$. In order to compute this velocity, denote the diffusion velocity of the interface normal to itself by $V_{d}$. The distance $\overline{A C}$ 
is then $V_{d} d t$. Now the cosine of the angle $C B A$ can be computed two ways:

$$
\cos C B A=\mathbf{n}_{i} \cdot\left(\mathbf{t}_{v} \times \mathbf{t}_{\omega}\right)=\overline{A C} / \overline{A B} .
$$

The unit vector normal to the interface is denoted by $\mathbf{n}_{i}$. Combining these relations gives the velocity of $\overline{A B}$ (which is in the direction $\mathbf{t}_{v} \times \mathbf{t}_{\omega}$ ):

$$
\frac{\overline{A B}}{d t}=\frac{V_{d}}{\mathbf{n}_{i} \cdot \mathbf{t}_{v} \times \mathbf{t}_{\omega}}\left(\mathbf{t}_{v} \times \mathbf{t}_{\omega}\right) \text {. }
$$

Next the distance $\overline{B B^{\prime}}$ is related to the velocity of point $A$ by two terms of a Taylor series:

$$
\overline{B B^{\prime}} / d t=\mathbf{v}_{B}=\mathbf{v}_{A}+\overline{A B} \cdot \nabla \mathbf{v}_{A} .
$$

Since $\overline{A B}$ is of order $d t$, the motion of $B$ with respect to $A$ drops out in the limit as $d t \rightarrow 0$. The final result for the velocity of the vortex line at the interface becomes

$$
\mathbf{v}_{\chi, i}=\frac{\overline{A D}}{d t}+\frac{\overline{B B^{\prime}}}{\overline{d t}}=\frac{V_{d}}{\mathbf{n}_{i} \cdot \mathbf{t}_{v} \times \mathbf{t}_{\omega}}\left(\mathbf{t}_{v} \times \mathbf{t}_{\omega}\right)+\mathbf{v}_{A}
$$

The velocity consists of the local fluid velocity plus the viscous diffusion velocity in the direction of the $\chi$-surface.

Since the $\mathcal{X}$-numbering system does not influence the vector components, a given vector decomposition allows many choices for the vortex-line histories. The choice made above requires the vortex lines to move with the fluid particles in the region of irrotational flow. This also determines the vortex-line motion in the vortical or turbulent region.

\section{Summary}

There are many ways to make a kinematic decomposition of a velocity field into irrotational and vortical components. A potential/complex-lamellar decomposition is made by requiring that the vortical component $\beta$ be perpendicular to the vorticity. The vector components may be represented in terms of Monge's potentials $\phi, \psi$ and $\chi$ such that $\alpha=\nabla \phi$ and $\beta=\psi \nabla \chi$. To make a complete and unique decomposition, three additional requirements are imposed. First, a specific direction for $\beta$ is chosen in the plane which is perpendicular to $\omega$. This is equivalent to choosing the direction $\nabla \chi$ or specifying the shape of the $\chi$-surfaces. The second requirement is to give reference values $\beta_{0}$ along any line which connects all the $\chi$-surfaces. These two requirements determine the vector components $\alpha$ and $\beta$, but not the potentials. The third requirement is to specify how numbers are assigned to the $\chi$-surfaces, including a time dependence. Numbering the $\chi$-surfaces also completely determines the $\psi$-surfaces.

There are two major physical interpretations which derive from the decomposition. The vortical component $\beta$ indicates the presence of vorticity, and vortex lines are identified and their movement marked by the intersections of $\psi$ - and $\chi$-surfaces.

Investigating the dynamic equations which govern incompressible flow leads to choosing the $\beta$-direction in the $\mathbf{v}, \omega$ plane. Such a choice breaks up the vortex force $\mathbf{v} \times \boldsymbol{\omega}$ into two collinear components $\alpha \times \omega$ and $\beta \times \omega$. It also defines the vortex force lines as the intersections of $\phi$ - and $\chi$-surfaces. Another simplification resulting from 
this choice is that the kinetic energy equation splits into two independent equations of similar form.

In applying the decomposition to the turbulent wall layer, the $\beta$-direction was taken to be in the $v, \omega$ plane, the reference value $\beta_{0}=0$ was assumed at the interface, and the motion of the $\mathcal{X}$-surface numbering system chosen such that interface vortex lines would follow the fluid particles. With these assumptions, the decomposition was shown to give a rigorous basis for dividing the shear layer into a completely potential, non-turbulent region and a turbulent region which contains both potential and vortical components. The decomposition also provides a rigorous basis for discussing the history of vortex lines even in a region where viscosity is important.

\section{REFERENCES}

ArIs, R. 1962 Vectors, Tensors and the Basic Equations of Fluid Mechanics. Prentice-Hall.

Batchelor, G. K. 1967 An Introduction to Fluid Dynamics. Cambridge University Press.

BraNd, L. 1947 Vector and Tensor Analysis. Wiley.

Clebsch, A. 1858 J. Math. 56, 1.

Corrsin, S. 1943 N.A.C.A. Wartime Rep. W.94.

HeLmhoLtz, H. $1857 J$. reine angew. Math. 55, 25.

Hussain, A. K. M. F. \& Reynolds, W. C. 1970 J. Fluid Mech. 41, 241.

INCE, E. L. 1956 Ordinary Differential Equations. Dover.

Kelloga, O. D. 1953 Foundations of Potential Theory. Dover.

Kelvin, Lord 1851 Phil. Trans. Roy. Soc. 141, 243.

Kovasznay, L. S. G., Kibens, V. \& Blackwelder, R. F. 1970 J. Fluid Mech. 41, 283.

LAMB, H. 1945 Hydrodynamics. Dover.

LaNDAHL, M. 'T. 1967 J. Fluid Mech. 29, 441.

LiBBy, P. 1975 J. Fluid Mech. 68, 273.

Phillips, H. B. 1933 Vector Analysis. Wiley.

Rrchardson, S. M. \& Cornish, A. R. H. 1977 J. Fluid Mech. 82, 309.

Stokes, G. G. 1851 Trans. Camb. Phil. Soc. 9, 1. (See also Papers, vol. 2, p. 243. Cambridge University Press.)

Tennekes, H. \& Lumley, J. L. 1972 A First Course in Turbulence. MIT Press.

Townsend, A. A. 1976 The Structure of Turbulent Shear Flow, 2nd edn. Cambridge University Press.

Truesdell, C. A. 1954 The Kinematics of Vorticity. Indiana University Press. 